\title{
Bioinformatic-based Identification of Genes Associated with Aortic Valve Stenosis
}

\author{
Chao Song, ${ }^{1}$ Shixiong Wei, ${ }^{1}$ Yunlong Fan, ${ }^{1}$ Shengli Jiang, ${ }^{1,2}$ \\ ${ }^{1}$ Medical School of Chinese PLA, Beijing, 100853, China; ${ }^{2}$ Department of Cardiovascular Surgery, the First Medical Centre of Chinese \\ PLA General Hospital, Beijing 100853, China
}

\section{ABSTRACT}

Background: Aortic valve stenosis (AS) disease is the most common valvular disease in developed countries. The pathology of AS is complex, and its main processes include calcification of the valve stroma and involve genetic factors, lipoprotein deposition and oxidation, chronic inflammation, osteogenic transition of cardiac valve interstitial cells, and active valve calcification. The aim of this study was to identify potential genes associated with AS.

Methods: Three original gene expression profiles (GSE153555, GSE12644, and GSE51472) were downloaded from the Gene Expression Omnibus (GEO) database and analyzed by GEO2R tool or 'limma' in R to identify differentially expressed genes (DEGs). Functional enrichment was analyzed using the ClusterProfiler package in R Bioconductor. STRING was utilized for the Protein-Protein Interaction (PPI) Network construct, and tissue-specific gene expression were identified using BioGPS database. The hub genes were screened out using the Cytoscape software. Related miRNAs were predicted in Targetscan, miWalk, miRDB, Hoctar, and TarBase.

Results: A total of 58 upregulated genes and 20 downregulated genes were screened out, which were mostly enriched in matrix remodeling and the immune system process. A module was thus clustered into by PPI network analysis, which mainly involved in Fc gamma R-mediated phagocytosis, Osteoclast differentiation. Ten genes (IBSP, NCAM1, MMP9, FCGR3B, COL4A3, FCGR1A, THY1, RUNX2, ITGA4, and COL10A1) with the highest degree scores were subsequently identified as the hub genes for AS by applying the CytoHubba plugin. And hsa-miR-1276 was finally identified as potential miRNA and miRNA-gene regulatory network was constructed using NetworkAnalyst.

Conclusions: Our analysis suggested that IBSP, NCAM1, MMP9, FCGR3B, COL4A3, FCGR1A, THY1, RUNX2, ITGA4, and COL10A1 might be hub genes associated with AS, and hsa-miR-1276 was potential miRNA. This result

\footnotetext{
August 23,2021; accepted September 27, 2021.

Correspondence: Dr. Shengli fiang, Department of Cardiovascular Surgery, the First Medical Centre of Chinese PLA General Hospital, No.28 Fuxing Rd, Beijing, 100853, China (e-mail:jiang_shengli301@163.com).
}

could provide novel insight into pathology and therapy of AS in the future.

\section{INTRODUCTION}

Aortic valve stenosis (AS) is the most common valvular disease in developed countries [Yadgir 2020]. As the aging population is dramatically rising worldwide, the incidence of AS is significantly increasing as well, which creates a huge burden on public health [Iung 2003; Nkomo 2006; Stritzke 2009; Coffey 2014]. As the age structures of global populations shift upward, calcific aortic valve stenosis (CAVS) is the most common valvular heart disease [Thériault 2018]. Other causes of AS include rheumatic fever, valvular degeneration, antiphospholipid syndrome, congenital aortic bicuspid valve and so on, with its primary pathological manifestation exhibited characterized by significantly increased afterload due to activity limitation of the aortic valve. While the hypertrophic response initially is compensatory to maintain heart function in the face of pressure overload (PO), pathological hypertrophy eventually becomes decompensatory, ultimately leading left heart failure [Carabello 2009; Kamperidis 2016].

The pathophysiology features of AS are mainly characterized by aberrant cell signaling pathways. Persistent activation of these aberrant cell signaling pathways leads to valve thickening known as sclerosis, which occurs simultaneously with the infiltration of $\mathrm{T}$ cells and macrophages, deposition of lipids, proteoglycans, and eventual calcification, causing the stenosis of the valve [Otto 1994; Bossé 2008; Coté 2013; Pasipoularides 2016]. It previously has been reported that abnormality of the signaling pathway involves genes involved in osteogenesis and calcium regulation, multiple matrix metalloproteinases, cytokines, chemokines, and collagen genes [Bossé 2009; Padang 2015; Guauque-Olarte 2016]. However, the pathogenesis of AS is a complex network that involves the regulation and mutual regulation of multiple signaling pathways. Current studies on biomarkers may be insufficient, and the differentially expressed gene (DEG) results may be inconsistent because of the complicated molecular regulatory mechanism of AS. Therefore, more effort should be invested toward the identification and understanding of novel biomarkers and specific targets of AS, which is considered the key to developing more effective diagnostic and therapeutic strategies. Recently, high-throughput gene chip and gene profiles increasingly have been used to screen differentially expressed genes (DEGs). Reanalyzing these data can 
provide new insights into current research on AS, which means bioinformatics methods could widely be used to find molecular changes in the occurrence and development of AS and are effective ways to explore the pathogenesis of diseases.

In this study, three original gene expression profiles (GSE153555, GSE12644, and GSE51472) were downloaded from the Gene Expression Omnibus (GEO) database. DEGs were first screened based on the above three datasets. Subsequently, integrated bioinformatics analyses, including Gene Ontology (GO) term analysis, Kyoto Encyclopedia of Genes and Genomes (KEGG) pathway enrichment analysis, protein-protein interaction (PPI) construction, the identification of hub genes and potential miRNA, were performed.

\section{METHODOLOGY}

Collection of the dataset: The NCBI-GEO database is a free and public database containing gene profiles. Aortic stenosis was used as the keyword to search the GEO database, then three original gene expression profiles (GSE153555, GSE12644, and GSE51472) were downloaded from the Gene Expression Omnibus (GEO) database. The inclusion criteria for the above gene expression profiles were set as follows: (1) the tissue samples were obtained from human AS tissues and normal tissues, and (2) the number of samples in each dataset was more than 10.

Identification of DEGs: GEO2R is an interactive web tool that can compare and analyze two different groups of samples under the same experimental conditions [Barrett 2013]. Using the GEO2R tool, we identified the differentially expressed genes in AS tissues and normal tissues. For GSE153555, according to the purpose of the study, we chose only the sample of stenotic aortic valves in this dataset as the study subjects. Gene expression data were first converted to TPM by normalizing FPKM values by the total FPKM in each tissue, then differential expression analysis was performed using limma ( $\mathrm{R}$ package limma) to identify individual genes demonstrating enrichment. Genes with Ilog [fold-change (FC)] $\mathrm{I}>0.5$ and $P<0.05$ were considered to be DEGs.

Among these DEGs from the three datasets, only DEGs that commonly appeared in all three datasets (intersection set) were considered as the significant DEGs. These were selected for subsequent analysis. And the upregulated and downregulated genes separately were analyzed.

Functional enrichment analysis of DEGs: To explore the potential function of DEGs in patients with AS, DEGs were subjected to GO enrichment analysis and Kyoto Encyclopedia of Genes and Genomes (KEGG) analysis to determine the associated biological process (BP), molecular function (MF), and cellular component (CC) terms using the Then, GO and KEGG pathway analyses of the DEGs were performed by DAVID [Yu 2012]. The data were loaded into NetworkAnalyst (https://www. networkanalyst.ca/) for re-analysis. This is a visual analytics platform for comprehensive gene expression profiling.

The top 10 items of the cellular component (CC), biological process (BP), and molecular function (MF) categories and KEGG pathways were then sorted and presented in the form of bubble maps. These bubble plots were drawn using the ClusterProfiler R package based on $P$-value $(<0.05$ was considered statistically significant) through the statistical software R (version 4.0.5).

Protein-Protein Interaction (PPI) Network Analysis: The protein-protein interaction (PPI) network was constructed by submitting a DEG list to the STRING (version 11.0; https://string-db.org/), which is an online database of known and predicted protein-protein interactions [Szklarczyk 2019]. To screen AS-related genes, DEGs were mapped into the Search Tool for the Retrieval of Interacting Genes database to predict PPI pairs with a combined score $>0.4$. The PPI networks were visualized using Cytoscape software (Version 3.6.1) [Shannon 2003], the plug-in Molecular Complex Detection (MCODE) was performed to screen modules of the PPI network with the threshold set as follows: MCODE scores $>10$.

Identification of hub genes and the tissue-specific expression: The hub gene is a class of genes that play a critical role in biological processes, and the regulation of other genes in related pathways is often regulated by these genes. Nodes with a degree of interaction $\geq 10$ in PPI Network Analysis were considered as hub genes. We thus used the online resource BioGPS (http://biogps.org), which was considered the functional localizer for the gene expression to analyze the tissue-specific expression of the DEGs.

Identification of the miRNA associated with AS and potential target genes: To identity the miRNA associated with AS, DEGs subsequently were subjected to Targetscan (http://www.targetscan.org/vert_71/), miWalk (http://mirwalk.umm.uni-heidelberg.de/), miRDB (http://mirdb.org/), Hoctar (https://hoctar.tigem.it/), and TarBase (https://www. tarbase.com/) databases, respectively, to screen miRNA, which could regulate mRNA expression in progression of AS. And the

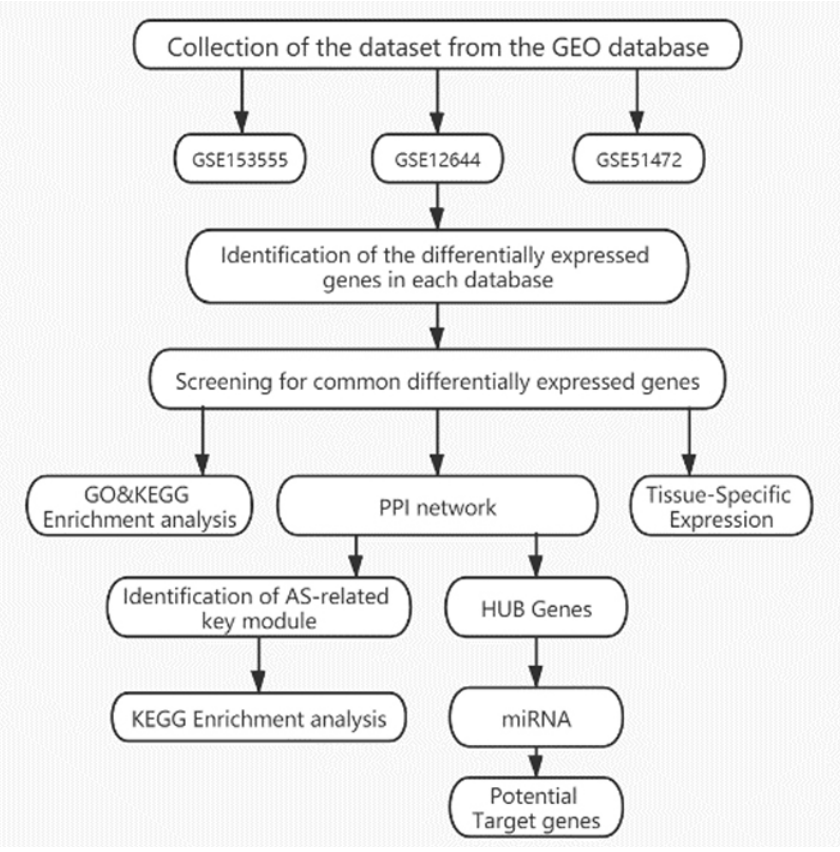

Figure 1. Flow chart of data preparation, processing, and analysis. 
Table 1. A summary of aortic valve stenosis microarray datasets from different GEO datasets

\begin{tabular}{llccc}
\hline No. of gene set & Type & Source & Case/Control & Platform \\
\hline GSE153555 & mRNA & Stenotic aortic valve & $10 / 10$ & GPL16791 \\
GSE12644 & mRNA & Stenotic aortic valve & $10 / 10$ & GPL570 \\
GSE51472 & mRNA & Stenotic aortic valve & $5 / 5$ & GPL570
\end{tabular}

Table 2. Differentially expressed genes identified by $R$

\begin{abstract}
DEGs Genes
Upregulated

Downregulated
\end{abstract}

\section{RESULTS}

DEG Screening: Three expression profiles (GSE153555, GSE12644, and GSE51472) were obtained from the GEO database. Among these profiles, GSE12644 and GSE51472 were based on GPL570 (Affymetrix Human Genome U133 Plus 2.0 Array), and GSE53555 was based on GPL16791 (Illumina HiSeq 2500). GSE153555 and GSE12644 contained 20 clinical samples separately: 10 case and 10 control samples, and GSE51472 contained 5 case and 5 control samples. The specific details of the above datasets are presented in Table 1.

A total of 78 DEGs between AS and control were finally screened out by R software, according to the above criteria, including 58 upregulated genes and 20 downregulated genes. (Table 2) (Figure 2)

Functional enrichment analysis of DEGs: A total of 58 upregulated genes and 20 downregulated genes were analyzed by DAVID software. Seventeen enriched GO terms and 6 KEGG pathways were enriched in DEGs between AS and normal aortic valve tissue $(P<0.05$ and $\mathrm{Q}$-value $<0.2)$. The top 5 significant terms from the GO enrichment analysis showed that in the BP category, the upregulated DEGs were involved in extracellular matrix organization, extracellular structure organization, endoderm development, leukocyte migration, and regulation of leukocyte cell-cell adhesion. Whereas the downregulated DEGs were significantly involved in the negative regulation of stress
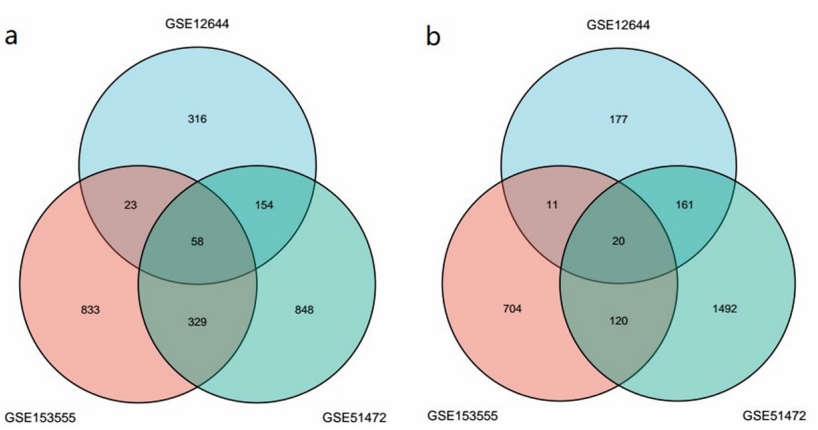

Figure 2. Venn diagram for overlapping differentially expressed genes (DEGs) based on datasets. The intersection of upregulated (a) and downregulated(b) DEGs was identified from the three datasets, namely, GSE153555, GSE12644 and GSE51472.

fiber assembly, negative regulation of actin filament bundle assembly, synapse organization, synapse assembly and regulation of filopodium assembly. For the CC category, the downregulated DEGs were correlated with axon part, dendritic spine, neuronal cell body, distal axon and rough endoplasmic reticulum. For the MF category, the upregulated DEGs were enriched in immunoglobulin binding, IgG binding, metalloendopeptidase activity, extracellular matrix binding, and collagen binding. Whereas the downregulated DEGs were related to flavin adenine dinucleotide binding, acyl-CoA dehydrogenase activity, sodium-potassium exchanging ATPase activity, potassium-transporting ATPase activity and potassium ion binding.

For KEGG pathway enrichment analysis, the top five significant KEGG pathways of the upregulated DEGs included Leishmaniasis, rheumatoid arthritis, hematopoietic cell lineage, systemic lupus erythematosus, and inflammatory bowel disease. Whereas the significant KEGG pathways of the downregulated DEGs included tyrosine metabolism, 
Table 3. Top 20 enriched terms for differentially expressed genes in AS

\begin{tabular}{|c|c|c|c|c|c|}
\hline hsa05140 & pathway_KEGG & Leishmaniasis & $5 / 42$ & 0.004852319 & $\begin{array}{c}\text { FCGR3B/HLA-DOB/FCGR1A/CR1/ } \\
\text { ITGA4 }\end{array}$ \\
\hline hsa00380 & pathway KEGG & Tryptophan metabolism & $3 / 42$ & 0.03632831 & TDO2/AOX1/MAOA \\
\hline hsa05323 & pathway KEGG & Rheumatoid arthritis & $4 / 42$ & 0.03632831 & CXCL5/MMP1/LTB/HLA-DOB \\
\hline hsa04640 & pathway KEGG & Hematopoietic cell lineage & $4 / 42$ & 0.03632831 & HLA-DOB/FCGR1A/CR1/ITGA4 \\
\hline GO:0043062 & geneontology_Biological_Process & $\begin{array}{l}\text { extracellular structure } \\
\text { organization }\end{array}$ & $12 / 71$ & 4.74E-05 & $\begin{array}{c}\mathrm{IBSP} / \mathrm{COL} 10 \mathrm{~A} 1 / \mathrm{MMP} 9 / \mathrm{COL} 11 \mathrm{~A} 1 / \\
\text { MMP1 }\end{array}$ \\
\hline GO:0030198 & geneontology_Biological_Process & $\begin{array}{l}\text { extracellular matrix orga- } \\
\text { nization }\end{array}$ & $11 / 71$ & $5.08 \mathrm{E}-05$ & $\begin{array}{c}\mathrm{IBSP} / \mathrm{COL} 10 \mathrm{~A} 1 / \mathrm{MMP} 9 / \mathrm{COL} 11 \mathrm{~A} 1 / \\
\text { MMP1 }\end{array}$ \\
\hline GO:0033267 & geneontology_Cellular_Component & axon part & $7 / 73$ & 0.014782528 & $\begin{array}{c}\text { STMN2/THY1/ITGA4/GNRH1/ } \\
\text { PPP1R9A }\end{array}$ \\
\hline GO:0030426 & geneontology_Cellular_Component & growth cone & $5 / 73$ & 0.014782528 & $\begin{array}{c}\text { STMN2/THY1/ITGA4/PPP1R9A/ } \\
\text { GPM6A }\end{array}$ \\
\hline GO:0030427 & geneontology_Cellular_Component & site of polarized growth & $5 / 73$ & 0.014782528 & $\begin{array}{c}\text { STMN2/THY1/ITGA4/PPP1R9A/ } \\
\text { GPM6A }\end{array}$ \\
\hline GO:0150034 & geneontology_Cellular_Component & distal axon & $6 / 73$ & 0.014782528 & $\begin{array}{c}\text { STMN2/THY1/ITGA4/GNRH1/ } \\
\text { PPP1R9A }\end{array}$ \\
\hline GO:0019865 & geneontology_Molecular_Function & immunoglobulin binding & $4 / 68$ & 0.000348675 & FCGR3B/FCGR1B/FCGR1A/MS4A2 \\
\hline GO:0030020 & geneontology_Molecular_Function & $\begin{array}{l}\text { extracellular matrix struc- } \\
\text { tural constituent conferring } \\
\text { tensile strength }\end{array}$ & $3 / 68$ & 0.014863511 & COL10A1/COL11A1/COL4A3 \\
\hline
\end{tabular}

tryptophan metabolism, drug metabolism, aldosterone synthesis and secretion, and AGE-RAGE signaling pathway. (Table 3) (Figure 3)

Protein-Protein Interaction (PPI) Network Analysis: To screen for crucial genes associated with AS, DEGs were mapped into the Search Tool for the Retrieval of Interacting Genes database (STRING; Version 11.0) to predict PPI pairs with a combined score $>0.4$. The PPI networks were visualized using Cytoscape software (Version 3.6.1).

The PPI network of the DEGs in AS was constructed based on the information obtained from the STRING database. When 78 DEGs were submitted to the STRING database, as illustrated in Figure 4, 74 of 78 genes were mapped into the PPI network. (Figure 4) The PPI network included 59 nodes and 23 edges, and its PPI enrichment $P$-value was 3.08e-10.

Then, the significant modules were identified via the MCODE plugin. One functional clusters of modules with three upregulated gene (FCGR1A, FCGR1B, and FCGR3B) and one downregulated gene (NCAM1) were identified. As illustrated in Figure 5, the MCODE score of the functional cluster of the module was 4 , which consisted of 4 nodes and 6 edges. (Figure 5) The enrichment analysis of that module indicated that these genes mainly were involved in Leishmaniasis, Fc gamma R-mediated phagocytosis, osteoclast differentiation, and systemic lupus erythematosus.

Identification of hub genes and the tissue-specific expression: Ten genes (IBSP, NCAM1, MMP9, FCGR3B, COL4A3, FCGR1A, THY1, RUNX2, ITGA4, and COL10A1) 
a

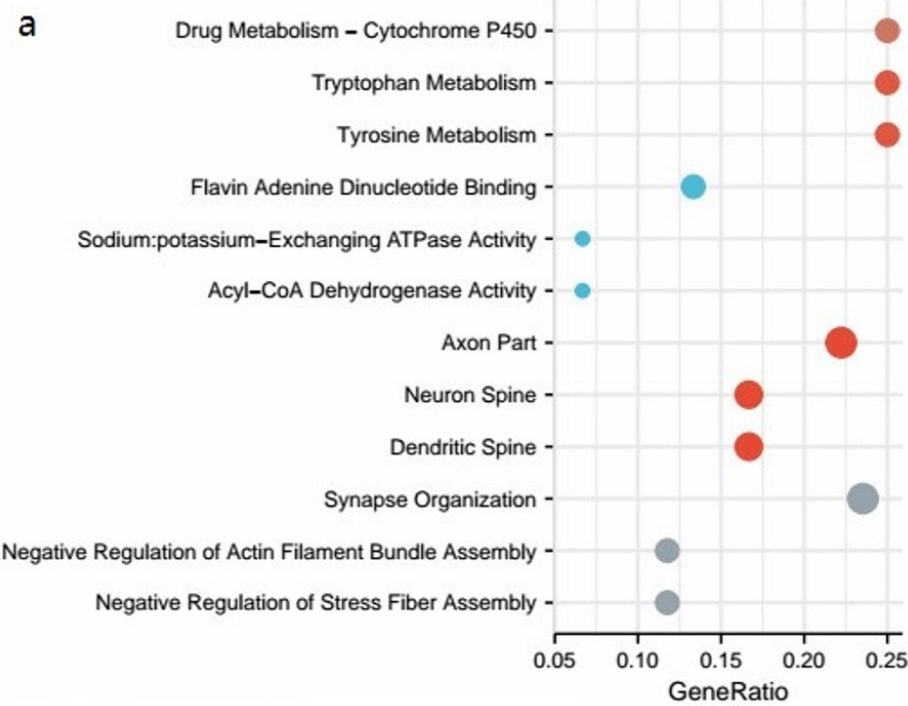

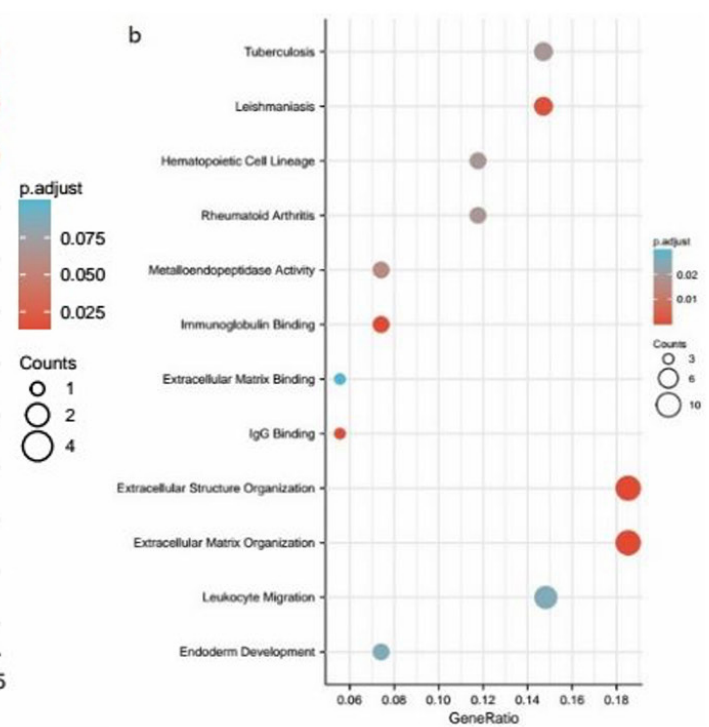

Figure 3. Biological process, molecular function and cellular component related GO terms identification result according to combined score. Bubble map for GO and KEGG pathway analyses of upregulated(a) and downregulated(b) DEGs.

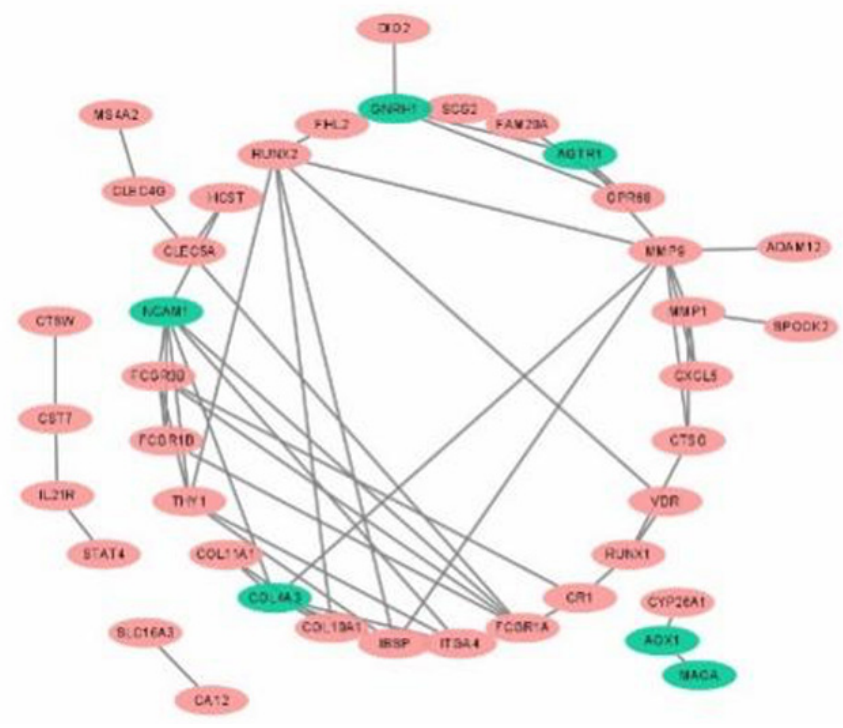

Figure 4. Protein-protein interactions (PPIs) network for identified common differentially expressed genes.

with the highest degree scores were identified as the hub genes for AS by applying the CytoHubba plugin. And furthermore, we identified 72 genes that were expressed in a specific tissue or organ system using BioGPS. The most highly tissue-specific expression system was the immune system $(29 \%, 21 / 72)$. This may indicate that immune-related pathways play an important role in the progression of AS. (Table 4) (Figure 6)

Identification of the miRNA associated with AS and potential target genes: microRNAs (miRNAs) are short non-coding RNAs that are involved in post-transcriptional regulation of gene expression in multicellular organisms by affecting both

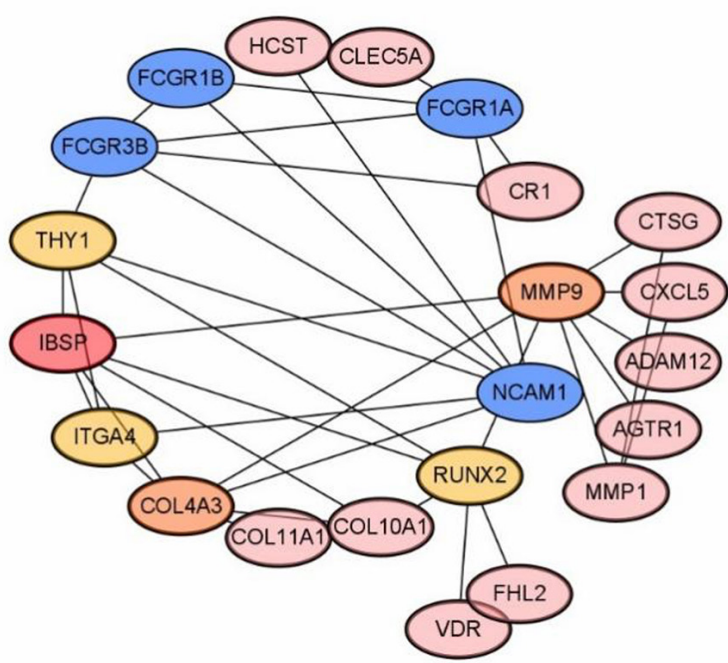

Figure 5. Module analysis network obtained from PPIs network. FCGR1A, FCGR1B, FCGR3B and NCAM1 are highlighted in blue. The network represents highly interconnected regions of the PPIs network which consisted of 4 nodes and 6 edges.

the stability and translation of mRNAs. The mature miRNA is incorporated into an RNA-induced silencing complex (RISC), which recognizes target mRNAs through imperfect base pairing with the miRNA and most commonly results in translational inhibition or destabilization of the target mRNA. We finally identified a miRNA (hsa-miR-1276) after performing intersection. We thus predicted potential NCAM1 as the target gene regulated by hsa-miR-1276 using NetworkAnalyst and construct the miRNA-gene regulatory network. Overall, the regulatory network could help to clarify the interaction between miRNAs and TG in AS progression. (Figure 7) (Figure 8) 
Table 4. Tissue-specific expressed genes identified by BioGPS

\begin{tabular}{ll}
\hline System & Genes \\
\hline Hematologic/Immune & IL21R, ITGA4, DTL, IFITM10, LTB, FCMR, HLA-DOB, FCGR1B, FCGR1A, CLEC5A, CR1, VDR, RASGRP1, MYO1G, CST7, CTSW, \\
& STAT4, SPOCK2, RGS13, RUNX1, MS4A, MMP9, CTSG, KCNJ15, FCGR3B \\
Neurologic & TMEM158, GPM6A, STMN2, CYP26A1, NCAM1, PCDH11Y, PCDH11X, SCG2, CADM1, PPP1R9A, COL4A3, FAM169A, THY1, \\
& CADM2, CNKSR2, ATP1A2 \\
Circulatory & IBSP, RUNX2, GNRH1, VMO1, FHL2 \\
Digestive & ADAMDEC1, CLEC4G, TMEM163, ADTRP, TDO2 \\
Other & COL11A1, FAM20A, AOX1, CA12, ITLN1, ADAM12, MAOA, AGTR1, STEAP1, MYOC, SYTL2, GPR68, CXCL5, MMP1, SLC16A3, \\
& DIO2, ACADL, COL10A1, LRRC15, HAND2-AS1, APELA, TSPAN11, LINC01013, GALNT5, HCST, TPTE2P
\end{tabular}

\section{DISCUSSION}

Aortic stenosis is the most common valvular disease in middle-aged and elderly people worldwide [Guauque-Olarte 2016; Yadgir 2020]. It increasingly has become a serious social problem, especially in developed countries. However, no drug or medical treatment has proven to be effective in reversing or delaying the progression of aortic stenosis [Nishimura 2017]. Aortic valvuloplasty or transcatheter aortic valve replacement currently is an effective treatment for AS. However, it still is not suitable for all patients as an invasive treatment. In recent years, with the development of the next generation sequencing and chip array techniques combined with advances in bioinformatics, a great deal of data has been accumulated in relation to various physiological or pathological conditions and provide an integrative, standardized, and comprehensive platform to facilitate the identification of DEGs and thus infer possible therapeutic options and molecular mechanisms of prognosis [Altman 2016].

In this study, three GEO datasets, namely, GSE153555, GSE12644 and GSE51472, were selected to screen DEGs. Applying the GEO2R tool and R software, the intersection of 78 DEGs was identified, including 58 upregulated DEGs and 20 downregulated DEGs. Then, GO and KEGG pathway analyses of the DEGs were performed by DAVID. The rest of the research study is continued with the analysis of PPIs, potential miRNA.

AS is a chronic inflammatory disease in which all stages of sterile inflammation are accompanied by extensive immune cell infiltration (e.g. macrophages, T cells and mast cells) [George 1998; Wu 2007; Carità 2016; Kostyunin 2019; Fan 2020; Goody 2020; Kostyunin 2020]. The pathology of AS is complex, and its main processes include calcification of the valve stroma and involve genetic factors, lipoprotein deposition and oxidation, chronic inflammation, osteogenic transition of cardiac valve interstitial cells, and active valve calcification [Ghaisas 2000; Lindman 2016]. AS caused by calcification progression also was marked by an emergence of smooth muscle cell activation, inflammation, and calcification-related pathways. In diseaseprone fibrosis, overrepresented proteins are functionally annotated as inflammatory responses and calcification pathways, and fibrosis-derived intervalvular stromal cells exhibit greater calcification potential than cells derived from the ventricles [Schlotter 2018]. Multiple dysfunctional immune cells and proinflammatory cytokines act synergistically to cause extracellular matrix remodeling and calcification [Nagy 2011].

The GO enrichment analysis indicated that the extracellular matrix organization, extracellular structure organization, eukocyte migration and regulation of leukocyte cell-cell adhesion, immunoglobulin binding, IgG binding, metalloendopeptidase activity, extracellular matrix binding and collagen binding were significantly enriched. These invading immune cells release pro-fibrotic and pro-inflammatory cytokines and induce high concentrations of chemokines to interact with corresponding receptors on the surface of monocytes/macrophages, leading to cell activation and recruitment to the site of infection [Michaud 2013]. This is partially consistent with the results reported in the previous literature [Guauque-Olarte 2016].

Similarly, The KEGG pathway results showed that inflammatory disease, amino acid metabolism, drug metabolism, aldosterone synthesis and secretion, and AGE-RAGE signaling pathway significantly were enriched. The enrichment analysis results are consistent with previous findings that calcification of valve matrix components is an important contributor in the progression of aortic stenosis and shares a common pathology with endothelial injury, lipoprotein deposition, chronic inflammation, and matrix remodeling [de Oliveira Sá 2020; Kostyunin 2020]. The important role for abnormal activation of the Renin-angiotensin-aldosterone system in the progression of AS has been identified [Wasywich 2006; Cui 2017; Ibarrola 2018; Sen 2020]. Furthermore, abnormal tyrosine metabolism also may somehow play a regulatory role in the progression of AS [Lok 2013; Thakur 2013; Gottschalk 2018]. These results also provided significant clues to studying molecular interactions in the progression of AS. Indeed, many studies have indicated that metabolic pathways and the cell cycle are highly associated with the progression of AS [Teng 2020; Zhao 2020; Sun 2021].

As the most prominent section of the study, analysis of modules, hub gene detection and drug identification thoroughly depend on the PPIs network. The PPI network included 59 nodes and 23 edges, and its PPI enrichment $P$-value was $3.08 \mathrm{e}$ 10. Ten genes (IBSP, NCAM1, MMP9, FCGR3B, COL4A3, FCGR1A, THY1, RUNX2, ITGA4, and COL10A1) with the highest degrees of connectivity in the PPI network were identified. Subsequently, the significant modules were identified via the MCODE plugin. One functional cluster of modules with three upregulated gene (FCGR1A, FCGR1B, and FCGR3B) and one downregulated gene (NCAM1) were identified. The 


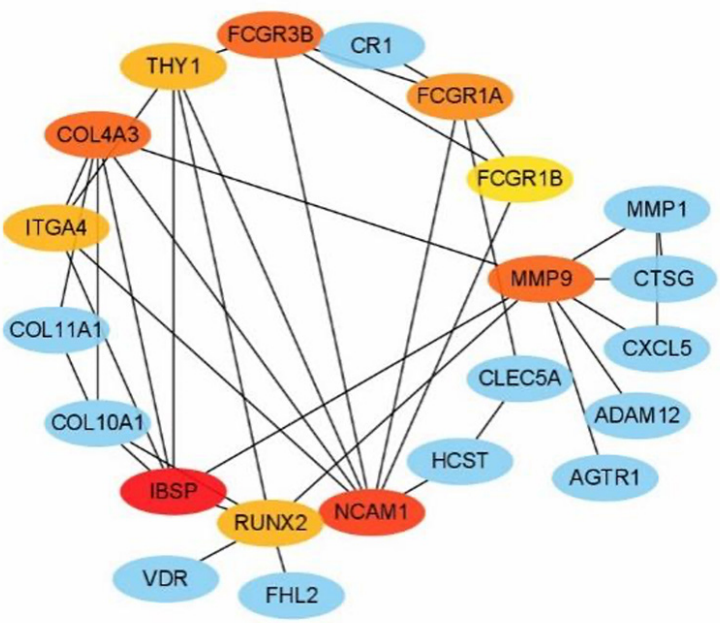

Figure 6. Detection of hub genes from the PPIs network of common DEGs. The highlighted 10 genes are BSP, NCAM1, MMP9, FCGR3B, COL4A3, FCGR1A, THY1, RUNX2, ITGA4, and COL10A1. These 10 genes are considered as hub genes, according to their degree value.

KEGG enrichment analysis of module 1 indicated that these genes were involved in Fc gamma R-mediated phagocytosis, osteoclast differentiation, Leishmaniasis. It has been confirmed in previous studies that the underlying pathophysiology of AS is complex and involves endothelial dysfunction, immune cell infiltration, myofibroblastic and osteoblastic differentiation, and, subsequently, calcification [Caira 2006; Shao 2006; Zhao 2010; Miller 2011; Goody 2020]. In this study, the expression levels of all hub genes were dysregulated in AS tissues compared with normal tissues, meaning that these genes may be crucial to progression in AS.

ITGA4 could encode a member of the integrin alpha chain family of proteins that function in cell surface adhesion and signaling. Susceptibility to coronary atherosclerosis and prognosis of disease progression were found to be associated with ITGA4, involved in metabolism of the extracellular matrix and processes of fibrogenesis. Furthermore, the MiR-30s family could also inhibit the proliferation and apoptosis in human coronary artery endothelial cells through targeting the 3'UTR Region of ITGA4 and PLCG1 [Ma 2016]. Considering that AS shares a similar pathway with atherosclerotic disease, it is necessary to ascertain the exact role of ITGA4 in progression of AS.

This RUNX2-encoded protein is essential for osteoblastic differentiation and skeletal morphogenesis and acts as a scaffold for nucleic acids and regulatory factors involved in skeletal gene expression [Garg 2005]. Osteogenic activity in the aortic valve is under the control of NOTCH1, which further promotes extracellular matrix remodeling by regulating the expression of RUNX2, a critical osteoinductive gene [Hadji 2016]. The upregulation of RUNX2 was observed in ongoing active processes of osteogenesis in inflamed valves using multimodality molecular imaging [Aikawa 2007].

The THY1-encoded protein is involved in cell adhesion and cell communication in numerous cell types. It can further

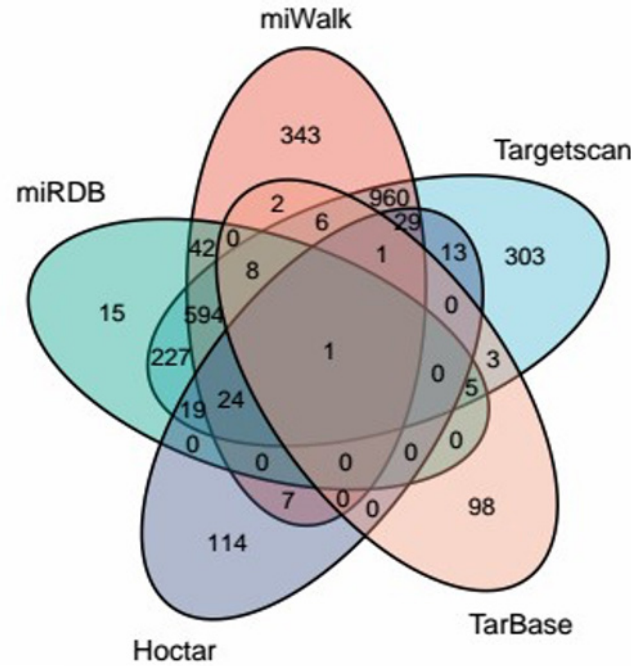

Figure 7. Venn diagram for overlapping miRNAs based on five datasets.

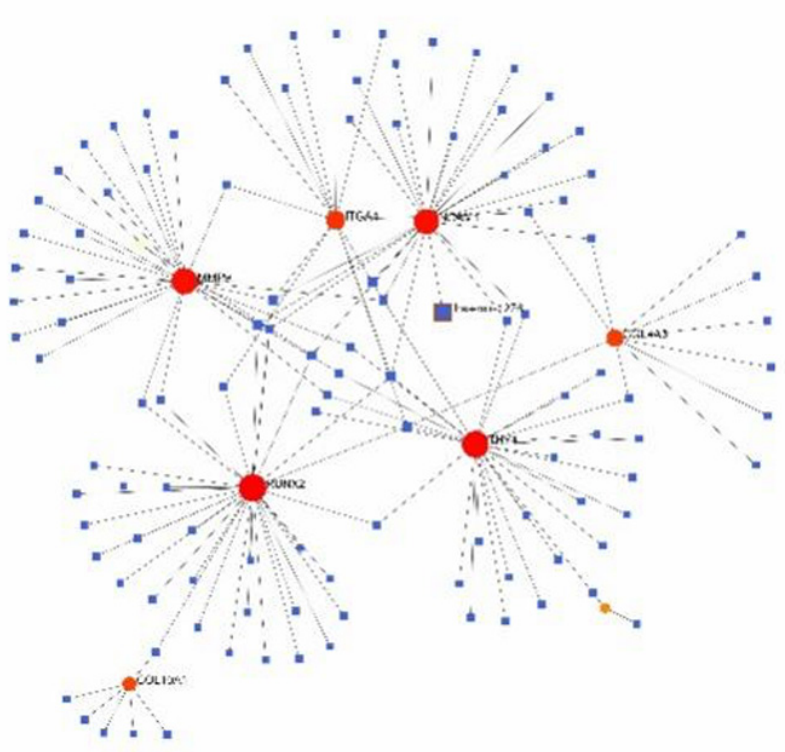

Figure 8. miRNA-gene regulatory network.

differentiate into cardiac fibroblasts (CFs) and smooth muscle cells (SMCs) [Gambardella 2019]. A significant upregulation of THY1 was identified in patients, who had undergone tissue-engineered heart valve replacement, and the mesenchymal stem cell subfraction significantly proliferated [VeDepo 2018].

The IBSP-encoded proteins are major structural protein of the bone matrix. This protein binds to calcium and hydroxyapatite via its acidic amino acid clusters, and mediates cell attachment through an RGD sequence that recognizes the vitronectin receptor [Kerr 1993; Ferreira 2021]. Previous studies indicated that calcified valves present distinct signs of active bone remodeling, and upregulation of IBSP plays a significant role in the progression of calcified AS [Pohjolainen 
2008; Greene 2020]. In addition, IBSP has proven to be highly associated with atherosclerotic diseases [Olesen 2007].

Proteins of the matrix metalloproteinase 9 (MMP9) family are involved in the breakdown of extracellular matrix in normal physiological processes and tissue remodeling, as well as in disease processes, such as arthritis and metastasis. MMP9 was identified to be involved in remodeling of the extracellular matrix and the progression of AS [Soini 2001; Gharibeh 2018; Swaminathan 2019; Urban 2019].

AS the major structural component of basement membranes, COL4A3 was significantly overexpressed in heart. Previous studies indicated that overexpression of COL4A3 in the myocardium leads to left ventricular diastolic dysfunction as well as cardiac hypertrophy [Touchberry 2013; Yousefi 2019].

COL10A1 encodes the alpha chain of type $X$ collagen, a short chain collagen expressed by hypertrophic chondrocytes during endochondral ossification. Excessive chondrogenic proteoglycan accumulation and disruption of stratified extracellular matrix maintenance in the aortic valve leaflets are characteristics of myxomatous valve disease. Overexpression of COL10A1 would lead to excessive chondrogenic proteoglycan accumulation and disruption of stratified extracellular matrix maintenance in the aortic valve leaflets and further leads to chondrogenic differentiation of aortic valve interstitial cells as well as AS [Fang 2014; Greene 2020].

As the potential target gene regulated by hsa-miR-1276, NCAM1 was significantly overexpressed in the heart, and the encoded protein involved in the expansion of $\mathrm{T}$ lymphocytes, B lymphocytes and natural killer (NK) cells, which play an important role in immune surveillance. This protein plays a role in signal transduction by interacting with fibroblast growth factor receptors, $\mathrm{N}$-cadherin and other components of the extracellular matrix and by triggering signaling cascades involving FYN-focal adhesion kinase (FAK), mitogenactivated protein kinase (MAPK), and phosphatidylinositol 3-kinase (PI3K). NCAM1 was significantly upregulated in human heart samples from cardiomyopathy patients and NCAM1 protein levels correlated with the degree of TGF 1 activity in the human cardiac ventricle[Ackermann 2017]. Furthermore, NCAM1 also has been identified as a gene that plays a critical role in the replication pathway during the progression of coronary artery disease [Tur 2013; Ghosh 2015] and left ventricular wall remodeling [Arnett 2011].

We finally focused our attention on the FCGR family, including FCGR1A and FCGR3B, which plays an important role in immune cell activation, balanced immune protection, and immunopathology. The receptor of the FCGR family was recently identified as a major determinant of antibody-mediated natural killer $(\mathrm{NK})$ cell activation during immunization activities and could be the markers that reflect inflammatory or cytotoxic immune mechanisms contributing to its onset [Cybularz 2021]. In addition, the current study shows that patients with AS usually are accompanied by high expression of FCGR3A and increased levels of circulating intermediate monocytes [Pfluecke 2016; Hewing 2017, 2017; Mazur 2018; Pfluecke 2020], and furthermore, inflammatory cytokines would be produced, causing a cascade of inflammation which eventually become uncontrolled inflammation.
Further studies are required to study the relationship between these genes and AS and to subsequently elucidate the underlying mechanisms and provide possible therapeutic targets. Nevertheless, this predictive model is based on bioinformatics analysis and will have to be validated by further experimental studies to establish its clinical utility.

\section{CONCLUSION}

In this study, a total of 78 DEGs, including 58 upregulated DEGs and 20 downregulated DEGs in AS, were screened through integrated bioinformatic analysis. Ten genes of interest (IBSP, NCAM1, MMP9, FCGR3B, COL4A3, FCGR1A, THY1, RUNX2, ITGA4, and COL10A1) and the identifying key module were found by constructing a PPI network.

\section{REFERENCES}

Ackermann MA, Petrosino JM, Manring HR, Wright P, Shettigar V, Kilic A, et al. 2017. TGF- $\beta 1$ affects cell-cell adhesion in the heart in an NCAM1-dependent mechanism. J Mol Cell Cardiol. 112:49-57.

Aikawa E, Nahrendorf M, Sosnovik D, Lok VM, Jaffer FA, Aikawa M, et al. 2007. Multimodality molecular imaging identifies proteolytic and osteogenic activities in early aortic valve disease. Circulation. 115(3):377-386.

Altman R. 2016. Current Progress in Bioinformatics 2016. Brief Bioinform. 17(1):1.

Arnett DK, Meyers KJ, Devereux RB, Tiwari HK, Gu CC, Vaughan LK, et al. 2011. Genetic variation in NCAM1 contributes to left ventricular wall thickness in hypertensive families. Circ Res. 108(3):279-283.

Barrett T, Wilhite SE, Ledoux P, Evangelista C, Kim IF, Tomashevsky $M$, et al. 2013. NCBI GEO: archive for functional genomics data sets-update. Nucleic Acids Res. 41(Database issue):D991-995.

Bossé Y, Mathieu P, Pibarot P. 2008. Genomics: the next step to elucidate the etiology of calcific aortic valve stenosis. J Am Coll Cardiol. 51(14):1327-1336.

Bossé Y, Miqdad A, Fournier D, Pépin A, Pibarot P, Mathieu P. 2009. Refining molecular pathways leading to calcific aortic valve stenosis by studying gene expression profile of normal and calcified stenotic human aortic valves. Circ Cardiovasc Genet. 2(5):489-498.

Caira FC, Stock SR, Gleason TG, McGee EC, Huang J, Bonow RO, et al. 2006. Human degenerative valve disease is associated with up-regulation of low-density lipoprotein receptor-related protein 5 receptormediated bone formation. J Am Coll Cardiol. 47(8):1707-1712.

Carabello BA, Paulus WJ. 2009. Aortic stenosis. Lancet. 373(9667):956-966.

Carità P, Coppola G, Novo G, Caccamo G, Guglielmo M, Balasus F, et al. 2016. Aortic stenosis: insights on pathogenesis and clinical implications. J Geriatr Cardiol. 13(6):489-498.

Coffey S, Cox B, Williams MJ. 2014. The prevalence, incidence, progression, and risks of aortic valve sclerosis: a systematic review and metaanalysis. J Am Coll Cardiol. 63(25 Pt A):2852-2861.

Coté N, Mahmut A, Bosse Y, Couture C, Pagé S, Trahan S, et al. 2013. Inflammation is associated with the remodeling of calcific aortic valve disease. Inflammation. 36(3):573-581.

Cui L, Rashdan NA, Zhu D, Milne EM, Ajuh P, Milne G, et al. 2017. 
End stage renal disease-induced hypercalcemia may promote aortic valve calcification via Annexin VI enrichment of valve interstitial cell derivedmatrix vesicles. J Cell Physiol. 232(11):2985-2995.

Cybularz M, Wydra S, Berndt K, Poitz DM, Barthel P, Alkouri A, et al. 2021. Frailty is associated with chronic inflammation and pro-inflammatory monocyte subpopulations. Exp Gerontol. 149:111317.

de Oliveira Sá M, Cavalcanti L, Perazzo ÁM, Gomes R, Clavel MA, Pibarot P, et al. 2020. Calcific Aortic Valve Stenosis and Atherosclerotic Calcification. Curr Atheroscler Rep. 22(2):2.

Fan X, Peng J, Lei L, He J, Huang J, Zheng D, et al. 2020. Integrated analysis of immunocyte infiltration and differential gene expression in tricuspid aortic valve-associated thoracic aortic aneurysms. Ann Transl Med. 8(6):285

Fang M, Alfieri CM, Hulin A, Conway SJ, Yutzey KE. 2014. Loss of -catenin promotes chondrogenic differentiation of aortic valve interstitial cells. Arterioscler Thromb Vasc Biol. 34(12):2601-2608.

Ferreira SA, Young G, Jones JR, Rankin S. 2021. Bioglass/carbonate apatite/collagen composite scaffold dissolution products promote human osteoblast differentiation. Mater Sci Eng C Mater Biol Appl. 118:111393.

Gambardella L, McManus SA, Moignard V, Sebukhan D, Delaune A, Andrews S, et al. 2019. BNC1 regulates cell heterogeneity in human pluripotent stem cell-derived epicardium. Development. 146(24).

Garg V, Muth AN, Ransom JF, Schluterman MK, Barnes R, King IN, et al. 2005. Mutations in NOTCH1 cause aortic valve disease. Nature. 437(7056):270-274.

George J, Afek A, Gilburd B, Blank M, Levy Y, Aron-Maor A, et al. 1998. Induction of early atherosclerosis in LDL-receptor-deficient mice immunized with beta2-glycoprotein I. Circulation. 98(11):1108-1115.

Ghaisas NK, Foley JB, Briain DS, Crean P, Kelleher D, et al. 2000. Adhesion molecules in nonrheumatic aortic valve disease: endothelial expression, serum levels and effects of valve replacement. J Am Coll Cardiol. 36(7):2257-2262.

Gharibeh L, Komati H, Bossé Y, Boodhwani M, Heydarpour M, Fortier M, et al. 2018. GATA6 Regulates Aortic Valve Remodeling, and Its Haploinsufficiency Leads to Right-Left Type Bicuspid Aortic Valve. Circulation. 138(10):1025-1038.

Ghosh S, Vivar J, Nelson CP, Willenborg C, Segrè AV, Mäkinen VP, et al. 2015. Systems Genetics Analysis of Genome-Wide Association Study Reveals Novel Associations Between Key Biological Processes and Coronary Artery Disease. Arterioscler Thromb Vasc Biol. 35(7):1712-1722.

Goody PR, Hosen MR, Christmann D, Niepmann ST, Zietzer A, Adam M, et al. 2020. Aortic Valve Stenosis: From Basic Mechanisms to Novel Therapeutic Targets. Arterioscler Thromb Vasc Biol. 40(4):885-900.

Gottschalk BH, Blankenstein J, Guo L. 2018. Ochronosis of Mitral Valve and Coronary Arteries. Ann Thorac Surg. 106(1):e19-19e20.

Greene CL, Jaatinen KJ, Wang H, Koyano TK, Bilbao MS, Woo YJ. 2020. Transcriptional Profiling of Normal, Stenotic, and Regurgitant Human Aortic Valves. Genes (Basel). 11(7).

Guauque-Olarte S, Droit A, Tremblay-Marchand J, Gaudreault N, Kalavrouziotis D, Dagenais F, et al. 2016. RNA expression profile of calcified bicuspid, tricuspid, and normal human aortic valves by RNA sequencing. Physiol Genomics. 48(10):749-761.

Hadji F, Boulanger MC, Guay SP, Gaudreault N, Amellah S, Mkannez G, et al. 2016. Altered DNA Methylation of Long Noncoding RNA H19 in Calcific Aortic Valve Disease Promotes Mineralization by Silencing NOTCH1. Circulation. 134(23):1848-1862.
Hewing B, Au SC, Ludwig A, Ellerbroek R, van Dijck P, Hartmann L, et al. 2017. Severe Aortic Valve Stenosis in Adults is Associated with Increased Levels of Circulating Intermediate Monocytes. J Cardiovasc Transl Res. 10(1):27-34.

Hewing B, Ellerbroek R, Au SC, Stangl V, Dreger H, Laule M, et al. 2017. Levels of Circulating Intermediate Monocytes Decrease after Aortic Valve Replacement in Patients with Severe Aortic Stenosis. Thromb Haemost. 117(12):2346-2355.

Ibarrola J, Sadaba R, Martinez-Martinez E, Garcia-Peña A, Arrieta V, Alvarez V, et al. 2018. Aldosterone Impairs Mitochondrial Function in Human Cardiac Fibroblasts via A-Kinase Anchor Protein 12. Sci Rep. 8(1):6801

Iung B, Baron G, Butchart EG, Delahaye F, Gohlke-Bärwolf C, Levang OW, et al. 2003. A prospective survey of patients with valvular heart disease in Europe: The Euro Heart Survey on Valvular Heart Disease. Eur Heart J. 24(13):1231-1243

Kamperidis V, Delgado V, van Mieghem NM, Kappetein AP, Leon MB, Bax JJ. 2016. Diagnosis and management of aortic valve stenosis in patients with heart failure. Eur J Heart Fail. 18(5):469-481.

Kerr JM, Fisher LW, Termine JD, Wang MG, McBride OW, Young MF. 1993. The human bone sialoprotein gene (IBSP): genomic localization and characterization. Genomics. 17(2):408-415.

Kostyunin A, Mukhamadiyarov R, Glushkova T, Bogdanov L, Shishkova D, Osyaev N, et al. 2020. Ultrastructural Pathology of Atherosclerosis, Calcific Aortic Valve Disease, and Bioprosthetic Heart Valve Degeneration: Commonalities and Differences. Int J Mol Sci. 21(20).

Kostyunin AE, Yuzhalin AE, Ovcharenko EA, Kutikhin AG. 2019. Development of calcific aortic valve disease: Do we know enough for new clinical trials. J Mol Cell Cardiol. 132:189-209.

Lindman BR, Clavel MA, Mathieu P, Iung B, Lancellotti P, Otto CM, et al. 2016. Calcific aortic stenosis. Nat Rev Dis Primers. 2:16006.

Lok ZS, Goldstein J, Smith JA. 2013. Alkaptonuria-associated aortic stenosis. J Card Surg. 28(4):417-420.

Ma F, Li T, Zhang H, Wu G. 2016. MiR-30s Family Inhibit the Proliferation and Apoptosis in Human Coronary Artery Endothelial Cells Through Targeting the 3'UTR Region of ITGA4 and PLCG1. J Cardiovasc Pharmacol. 68(5):327-333.

Mazur P, Mielimonka A, Natorska J, Wypasek E, Gaw da B, Sobczyk D, et al. 2018. Lymphocyte and monocyte subpopulations in severe aortic stenosis at the time of surgical intervention. Cardiovasc Pathol. 35:1-7.

Michaud M, Balardy L, Moulis G, Gaudin C, Peyrot C, Vellas B, et al. 2013. Proinflammatory cytokines, aging, and age-related diseases. J Am Med Dir Assoc. 14(12):877-882.

Miller JD, Weiss RM, Heistad DD. 2011. Calcific aortic valve stenosis: methods, models, and mechanisms. Circ Res. 108(11):1392-1412.

Nagy E, Andersson DC, Caidahl K, Eriksson MJ, Eriksson P, FrancoCereceda A, et al. 2011. Upregulation of the 5-lipoxygenase pathway in human aortic valves correlates with severity of stenosis and leads to leukotriene-induced effects on valvular myofibroblasts. Circulation. 123(12):1316-1325.

Nishimura RA, Otto CM, Bonow RO, Carabello BA, Erwin JP 3rd, Fleisher LA, et al. 2017. 2017 AHA/ACC Focused Update of the 2014 AHA/ACC Guideline for the Management of Patients With Valvular Heart Disease: A Report of the American College of Cardiology/American Heart Association Task Force on Clinical Practice Guidelines. Circulation. 135(25):e1159-1159e1195. 
Nkomo VT, Gardin JM, Skelton TN, Gottdiener JS, Scott CG, Enriquez-Sarano M. 2006. Burden of valvular heart diseases: a population-based study. Lancet. 368(9540):1005-1011.

O\&\#39, Brien KD. 2006. Pathogenesis of calcific aortic valve disease: a disease process comes of age (and a good deal more). Arterioscler Thromb Vasc Biol. 26(8):1721-1728.

Olesen P, Nguyen K, Wogensen L, Ledet T, Rasmussen LM. 2007. Calcification of human vascular smooth muscle cells: associations with osteoprotegerin expression and acceleration by high-dose insulin. Am J Physiol Heart Circ Physiol. 292(2):H1058-1064.

Otto CM, Kuusisto J, Reichenbach DD, Gown AM, Brien KD. 1994. Characterization of the early lesion of 'degenerative' valvular aortic stenosis. Histological and immunohistochemical studies. Circulation. 90(2):844-853.

Padang R, Bagnall RD, Tsoutsman T, Bannon PG, Semsarian C. 2015. Comparative transcriptome profiling in human bicuspid aortic valve disease using RNA sequencing. Physiol Genomics. 47(3):75-87.

Pasipoularides A. 2016. Calcific Aortic Valve Disease: Part 1--Molecular Pathogenetic Aspects, Hemodynamics, and Adaptive Feedbacks. J Cardiovasc Transl Res. 9(2):102-118.

Pfluecke C, Berndt K, Wydra S, Tarnowski D, Barthel P, Quick S, et al. 2016. Atrial fibrillation is associated with high levels of monocyte-platelet-aggregates and increased CD11b expression in patients with aortic stenosis. Thromb Haemost. 115(5):993-1000.

Pfluecke C, Wydra S, Berndt K, Tarnowski D, Cybularz M, Jellinghaus S, et al. 2020. Mon2-monocytes and increased CD-11b expression before transcatheter aortic valve implantation are associated with earlier death. Int J Cardiol. 318:115-120.

Pohjolainen V, Taskinen P, Soini Y, Rysä J, Ilves M, Juvonen T, et al. 2008. Noncollagenous bone matrix proteins as a part of calcific aortic valve disease regulation. Hum Pathol. 39(11):1695-1701.

Schlotter F, Halu A, Goto S, Blaser MC, Body SC, Lee LH, et al. 2018. Spatiotemporal Multi-Omics Mapping Generates a Molecular Atlas of the Aortic Valve and Reveals Networks Driving Disease. Circulation. 138(4):377-393.

Sen J, Chung E, Neil C, Marwick T. 2020. Antihypertensive therapies in moderate or severe aortic stenosis: a systematic review and meta-analysis. BMJ Open. 10(10):e036960.

Shannon P, Markiel A, Ozier O, Baliga NS, Wang JT, Ramage D, et al. 2003. Cytoscape: a software environment for integrated models of biomolecular interaction networks. Genome Res. 13(11):2498-2504.

Shao JS, Cai J, Towler DA. 2006. Molecular mechanisms of vascular calcification: lessons learned from the aorta. Arterioscler Thromb Vasc Biol. 26(7):1423-1430.

Soini Y, Satta J, Määttä M, Autio-Harmainen H. 2001. Expression of MMP2, MMP9, MT1-MMP, TIMP1, and TIMP2 mRNA in valvular lesions of the heart. J Pathol. 194(2):225-231.

Stritzke J, Linsel-Nitschke P, Markus MR, Mayer B, Lieb W, Luchner A, et al. 2009. Association between degenerative aortic valve disease and longterm exposure to cardiovascular risk factors: results of the longitudinal population-based KORA/MONICA survey. Eur Heart J. 30(16):2044-2053.

Sun JY, Hua Y, Shen H, Qu Q, Kan JY, Kong XQ, et al. 2021. Identification of key genes in calcific aortic valve disease via weighted gene coexpression network analysis. BMC Med Genomics. 14(1):135.

Swaminathan G, Krishnamurthy VK, Sridhar S, Robson DC, Ning Y, Grande-Allen KJ. 2019. Hypoxia Stimulates Synthesis of Neutrophil
Gelatinase-Associated Lipocalin in Aortic Valve Disease. Front Cardiovasc Med. 6:156.

Szklarczyk D, Gable AL, Lyon D, Junge A, Wyder S, Huerta-Cepas J, et al. 2019. STRING v11: protein-protein association networks with increased coverage, supporting functional discovery in genome-wide experimental datasets. Nucleic Acids Res. 47(D1):D607-607D613.

Teng P, Xu X, Ni C, Yan H, Sun Q, Zhang E, et al. 2020. Identification of key genes in calcific aortic valve disease by integrated bioinformatics analysis. Medicine (Baltimore). 99(29):e21286.

Thakur S, Markman P, Cullen H. 2013. Choice of valve prosthesis in a rare clinical condition: aortic stenosis due to alkaptonuria. Heart Lung Circ. 22(10):870-872.

Thériault S, Gaudreault N, Lamontagne M, Rosa M, Boulanger MC, Messika-Zeitoun D, et al. 2018. A transcriptome-wide association study identifies PALMD as a susceptibility gene for calcific aortic valve stenosis. Nat Commun. 9(1):988.

Touchberry CD, Green TM, Tchikrizov V, Mannix JE, Mao TF, Carney BW, et al. 2013. FGF23 is a novel regulator of intracellular calcium and cardiac contractility in addition to cardiac hypertrophy. Am J Physiol Endocrinol Metab. 304(8):E863-873.

Tur MK, Etschmann B, Benz A, Leich E, Waller C, Schuh K, et al. 2013. The $140-\mathrm{kD}$ isoform of CD56 (NCAM1) directs the molecular pathogenesis of ischemic cardiomyopathy. Am J Pathol. 182(4):1205-1218.

Urban P, Rabajdová M, Špaková I, Sabol F, Mičková H, Lakatosová K, et al. 2019. Molecular recognition of aortic valve stenosis and regurgitation. Eur Rev Med Pharmacol Sci. 23(24):10996-11003.

VeDepo M, Buse E, Quinn R, Hopkins R, Converse G. 2018. Extended bioreactor conditioning of mononuclear cell-seeded heart valve scaffolds. J Tissue Eng. 9:2041731418767216.

Wasywich CA, Webster MW, Richards AM, Stewart RA. 2006. Coronary sinus and ascending aortic levels of aldosterone, angiotensin II, and B-type natriuretic peptide in patients with aortic stenosis and in patients with coronary heart disease. Am J Cardiol. 97(7):1068-1072.

Wu HD, Maurer MS, Friedman RA, Marboe CC, Ruiz-Vazquez EM, Ramakrishnan R, et al. 2007. The lymphocytic infiltration in calcific aortic stenosis predominantly consists of clonally expanded $\mathrm{T}$ cells. J Immunol. 178(8):5329-5339.

Yadgir S, Johnson CO, Aboyans V, Adebayo OM, Adedoyin RA, Afarideh M, et al. 2020. Global, Regional, and National Burden of Calcific Aortic Valve and Degenerative Mitral Valve Diseases, 1990-2017. Circulation. 141(21):1670-1680

Yousefi K, Irion CI, Takeuchi LM, Ding W, Lambert G, Eisenberg T, et al. 2019. Osteopontin Promotes Left Ventricular Diastolic Dysfunction Through a Mitochondrial Pathway. J Am Coll Cardiol. 73(21):2705-2718.

Yu G, Wang LG, Han Y, He QY. 2012. clusterProfiler: an R package for comparing biological themes among gene clusters. OMICS. 16(5):284-287.

Zhao Q, Wang X, Liu Y, He A, Jia R. 2010. NFATc1: functions in osteoclasts. Int J Biochem Cell Biol. 42(5):576-579.

Zhao X, Hou C, Xiao T, Xie L, Li Y, Jia J, et al. 2020. An interesting Mybpc3 heterozygous mutation associated with bicuspid aortic valve. Transl Pediatr. 9(5):610-618.

Zhou G, Soufan O, Ewald J, Hancock R, Basu N, Xia J. 2019. NetworkAnalyst 3.0: a visual analytics platform for comprehensive gene expression profiling and meta-analysis. Nucleic Acids Res. 47(W1):W234-234W241. 